\title{
Chemical biology makes an impact
}

\author{
Nature Chemical Biology's impact factor of 12.409 reflects the growing visibility and impact of research at the \\ interface of chemistry and biology.
}

$\mathbf{N}$ ature Chemical Biology has received its first impact factor of 12.409, based on 2006 citations of papers published from June 2005 through December 2005 (Thomson Scientific Journal Citation Reports, http://portal.isiknowledge.com). This premier impact factor positions Nature Chemical Biology not only as the leading chemical biology journal but also first among chemistry journals that publish original research related to biological science.

For the editorial team, Nature Chemical Biology's first impact factor was a welcome confirmation that the journal is achieving our stated aim "to create a top-tier international journal that reflects the diversity and excitement of chemical biology research" (Nat. Chem. Biol. 1, 3, 2005). In our first volume, which consists of seven issues, we published papers with authors from 18 countries, including several influential discoveries: 'necroptosis', a new cell death pathway (Nat. Chem. Biol. 1, 112-119, 2005), nitrite as a biological signaling molecule (Nat. Chem. Biol. 1, 290-297, 2005), an assay for promiscuous inhibition in drug discovery (Nat. Chem. Biol. 1, 146-148, 2005), and transcriptional gene inhibition by natural and artificial nucleic acids (Nat. Chem. Biol. 1, 210-215 and 216-222, 2005). According to Faculty of 1000 Biology (http://www.f1000biology.com), a website where scientists identify and rank current scientific papers, one quarter of the original research papers published in Nature Chemical Biology during 2005 received a ranking of "recommended" (18\%), "must read" (64\%), or "exceptional" (18\%). Nature Chemical Biology has also published a diverse collection of commissioned material such as Commentaries, News \& Views, Reviews and Perspectives, as well as editorial content such as editorials and research highlights, which are designed to introduce readers to current trends in the field and initiate discussion between chemists and biologists.

To achieve our goal of publishing the highest caliber original research and commentary in chemical biology, the editorial team has focused on identifying and developing interesting manuscripts that report important advances across chemistry and biology that are likely to appeal to a broad readership of chemical biologists. We are committed to helping authors improve their papers through the review process. Because of the interdisciplinary character of chemical biology, each paper is evaluated by a diverse set of referees whose expertise reflects the chemical and biological scope of the manuscript. For those papers that perform well at review, the editors advise authors as they revise their manuscripts. Of the papers that are published in the journal, most have undergone more than one cycle of review, which ensures the technical soundness and scientific completeness of each paper. We believe that our editorial and review processes identify the most important papers and make even the best papers more influential and understandable to the broad readership of the journal.
While we are decidedly pleased with our initial impact factor, we are equally concerned with the growing importance of numerical ranking systems for evaluating science and scientists. Throughout the world, the quality and importance of a scientific paper are often equated with the impact factor of the journal in which it is published. Yet awareness of impact factor data varies widely across academic disciplines. Informal discussions with chemical biologists suggest that biologists tend to be more aware of impact factor scores for specific journals than their chemistry counterparts, perhaps in part because of the broader range of these scores: whereas impact factors for biological journals can exceed 20 , chemical journals have traditionally peaked near 10 . Even though they may focus less on the numerical value of a journal's impact factor, chemists are quick to identify the top-ranked chemistry journals and to describe community pressures to publish in them. The publishing habits of chemists and biologists also vary: most chemists publish more papers per year than biologists at a similar stage of their careers, but papers from chemists tend to be shorter, on average. Taken together, the diverse publication habits across the biology and chemistry communities lead to differing perceptions and applications of impact factor information.

These cultural differences present challenges for scientists working in interdisciplinary fields such as chemical biology. Scientists are increasingly evaluated-by administrators, review committees and program officers - on the basis of the number of high-impact publications that they produce. We believe that impact factor should not be used as the primary criterion for evaluating scientists or their work, particularly in interdisciplinary fields. Given that chemists and biologists apply fieldspecific standards, it is imperative that chemical biology evaluators establish fair standards for assessing the 'quality' and 'impact' of research at the chemistry-biology interface. Certain quantitative metrics, such as impact factor, eigenfactor (www.eigenfactor.org) and h-index (Proc. Natl. Acad. Sci. USA 102, 16569-16572, 2005), may provide a useful starting point for evaluating scientific productivity; however, traditional approaches, which apply unbiased expert evaluation and examine the long-term citation record of individual papers, remain the most effective means of assessing scientists and the impact of their scholarship.

The strong premier of Nature Chemical Biology reflects the enhanced impact that chemical biology is having around the world. The emergence of chemical biology departments in academic, industrial and government sectors, the expansion of journals dedicated to chemical biology research, and the growing list of international conferences on the subject all emphasize that the field of chemical biology is on an upward trajectory. As this growth phase occurs, we are extremely gratified by the community's support of Nature Chemical Biology, and we look forward to enhancing the impact of chemical biology through the continued publication of the best original research and commentary across the chemistry-biology frontier. 